\title{
Neurosurgical versus endovascular treatment of spinal dural arteriovenous fistulas: a multicenter study of 195 patients
}

\author{
Keisuke Takai, MD, PhD, ${ }^{8}$ Toshiki Endo, MD, PhD, ${ }^{2}$ Takao Yasuhara, MD, PhD, ${ }^{13}$ \\ Toshitaka Seki, MD, PhD, ${ }^{1}$ Kei Watanabe, MD, PhD, ${ }^{3}$ Yuki Tanaka, MD, ${ }^{3}$ \\ Ryu Kurokawa, MD, PhD, ${ }^{4}$ Hideaki Kanaya, MD, PhD, ${ }^{4}$ Fumiaki Honda, MD, PhD, ${ }^{5}$ \\ Takashi Itabashi, MD, PhD, ${ }^{6}$ Osamu Ishikawa, MD, PhD, ${ }^{7}$ Hidetoshi Murata, MD, PhD, ${ }^{9}$ \\ Takahiro Tanaka, MD, ${ }^{9}$ Yusuke Nishimura, MD, PhD, ${ }^{10}$ Kaoru Eguchi, MD, ${ }^{10}$ \\ Toshihiro Takami, MD, PhD, ${ }^{11}$ Yusuke Watanabe, MD,11 Takeo Nishida, MD, PhD,12 \\ Masafumi Hiramatsu, MD, PhD, ${ }^{13}$ Tatsuya Ohtonari, MD, PhD, ${ }^{14}$ Satoshi Yamaguchi, MD, PhD, ${ }^{15}$ \\ Takafumi Mitsuhara, MD, PhD, ${ }^{15}$ Seishi Matsui, MD, PhD, ${ }^{16}$ Hisaaki Uchikado, MD, PhD, ${ }^{17}$ \\ Gohsuke Hattori, MD, PhD, ${ }^{17}$ Hitoshi Yamahata, MD, PhD, ${ }^{18}$ and Makoto Taniguchi, MD, PhD ${ }^{8}$
}

\begin{abstract}
1Department of Neurosurgery, Hokkaido University Hospital, Sapporo; ${ }^{2}$ Department of Neurosurgery, Kohnan Hospital, Sendai; ${ }^{3}$ Department of Orthopaedic Surgery, Niigata University Medical and Dental Hospital, Niigata; ${ }^{\circ}$ Department of Neurosurgery, Dokkyo Medical University Hospital, Tochigi; ${ }^{5}$ Department of Neurosurgery, Gunma University Hospital, Gunma; ${ }^{6}$ Department of Orthopaedic Surgery, Japanese Red Cross Narita Hospital, Chiba; ${ }^{7}$ Department of Neurosurgery, The University of Tokyo Hospital, Tokyo; ${ }^{2}$ Department of Neurosurgery, Tokyo Metropolitan Neurological Hospital, Tokyo; ' $D$ epartment of Neurosurgery, Yokohama City University Hospital, Yokohama; ${ }^{10}$ Department of Neurosurgery, Nagoya University Hospital, Nagoya;

${ }^{11}$ Department of Neurosurgery, Osaka City University Graduate School of Medicine, Osaka; ${ }^{2}$ Department of Neurosurgery, Osaka University Graduate School of Medicine, Osaka; ${ }^{13}$ Department of Neurosurgery, Okayama University Graduate School of Medicine, Okayama; ${ }^{14}$ Department of Spinal Surgery, Brain Attack Center, Ota Memorial Hospital, Hiroshima; ${ }^{15}$ Department of Neurosurgery, Hiroshima University Hospital, Hiroshima; ${ }^{16}$ Department of Neurosurgery, Ehime University Hospital, Ehime; ${ }^{17}$ Department of Neurosurgery, Kurume University Hospital, Fukuoka; and ${ }^{18}$ Department of Neurosurgery, Kagoshima University Hospital, Kagoshima, Japan
\end{abstract}

OBJECTIVE The purpose of the present study was to compare the treatment success rates of primary neurosurgical and endovascular treatments in patients with spinal dural arteriovenous fistulas (dAVFs).

METHODS Data from 199 consecutive patients with thoracic and lumbosacral spinal dAVFs were collected from 18 centers. Angiographic and clinical findings, the rate of initial treatment failure or recurrence by procedures, risk factors for treatment failure, complications, and neurological outcomes were statistically analyzed.

RESULTS Spinal dAVFs were frequently detected in the thoracic region (81\%), fed by a single feeder (86\%), and shunted into an intradural vein via the dura mater. The fistulous connection between the feeder(s) and intradural vein was located at a single spinal level in 195 patients (98\%) and at 2 independent levels in 4 patients (2\%). Among the neurosurgical $(n=145)$, and endovascular $(n=50)$ treatment groups of single dAVFs $(n=195)$, the rate of initial treatment failure or recurrence was significantly higher in the index endovascular treatment group $(0.68 \%$ and $36 \%)$. A multivariate analysis identified endovascular treatment as an independent risk factor with significantly higher odds of initial treatment failure or recurrence (OR 69; 95\% $\mathrm{Cl} 8.7-546)$. The rate of complications did not significantly differ between the two treatment groups (4.1\% for neurosurgical vs $4.0 \%$ for endovascular treatment). With a median follow-up of 26 months, improvements of $\geq 1$ point in the modified Rankin Scale (mRS) score and Aminoff-Logue gait and Aminoff-Logue micturition grades were observed in $111(56 \%), 121(61 \%)$, and $79(40 \%)$ patients, respectively. Independent risk factors for lack of improvement in the Aminoff-Logue gait grades were multiple treatments due to initial treatment failure or recurrence (OR 3.1) and symptom duration (OR 1.02).

ABBREVIATIONS AL = Aminoff-Logue; $A V=$ arteriovenous; $d A V F=$ dural AV fistula; ICG = indocyanine green; mRS = modified Rankin Scale; NBCA = N-butyl-cyanoacrylate.

SUBMITTED March 4, 2020. ACCEPTED June 29, 2020.

INCLUDE WHEN CITING Published online November 13, 2020; DOI: 10.3171/2020.6.SPINE20309. 
CONCLUSIONS Based on data obtained from the largest and most recently assessed multicenter cohort, the present study shows that primary neurosurgery is superior to endovascular treatment for the complete obliteration of spinal dAVFs by a single procedure.

https://thejns.org/doi/abs/10.3171/2020.6.SPINE20309

KEYWORDS spinal vascular malformations; spinal arteriovenous shunts; dural arteriovenous shunts; surgery; endovascular embolization; vascular disorders

$\mathrm{S}$ PINAL arteriovenous (AV) shunts are spinal vascular lesions in which one or more spinal arteries and veins are connected without an intervening blood capillary system. ${ }^{1,2}$ Spinal dural AV fistulas (dAVFs) are the most common type of spinal AV shunt and are characterized by an AV connection between radiculomeningeal arteries and an intradural vein on the inner surface of the spinal dura mater. ${ }^{3}$ The prognosis of patients with spinal $\mathrm{dAVFs}$ is poor if left untreated because venous hypertension leads to irreversible spinal cord infarction. ${ }^{4}$ There are two main treatments for patients with spinal dAVFs: neurosurgical and endovascular treatment. ${ }^{5,6}$ Neurosurgery is the gold standard treatment for dAVFs; however, spinal dAVFs have been increasingly treated with endovascular embolization ${ }^{7}$ due to rapid advances in endovascular techniques over the past 2 decades. ${ }^{8}$ Due to recent improvements in endovascular techniques and their priority over neurosurgery, it remains unclear whether spinal dAVFs need to be treated primarily with neurosurgical or endovascular treatment. To date, direct comparisons of treatment success rates between neurosurgical and endovascular treatments have rarely been conducted. ${ }^{9}$ Furthermore, $\mathrm{dAVF}$ recurrence and retreatment after treatment failure have not yet been examined in detail. ${ }^{10}$ The present study was conducted in a large multicenter cohort of dAVF patients to compare the rates of primary treatment success and the time of recurrence and success rates of retreatment between neurosurgical and endovascular treatment methods.

\section{Methods Ethics}

This study protocol was approved by the Institutional Review Board at the Tokyo Metropolitan Neurological Hospital (no. TS-H30-050) and participating centers. Since this was a retrospective and noninvasive study, the requirement for written informed consent from patients was waived. A public notice that provided information on this study was instead given on each center's website.

\section{Patients}

Consecutive patients with dAVFs in the thoracic and lumbosacral regions who were treated with neurosurgery and/or endovascular embolization between 2009 and 2018 were included. Treatments were indicated for symptomatic patients with spinal dAVFs. Patients were excluded if they had cervical, intradural, or epidural lesions; no angiographic data; or no long-term follow-up clinical data $(<$ 6 months). Angiographic and clinical data were collected from 18 centers in Japan using a standardized form (Fig. 1). Participating centers were selected from those certified by the Japan Neurosurgical Society, Neurospinal So- ciety of Japan, or Japanese Society for Spine Surgery and Related Research based on a recognized reputation in the treatment of spinal AV shunts. Among the 18 centers, neurosurgery is the first choice of treatment in 9 centers; endovascular treatment is the first choice in 4 centers; and the decision to perform surgery or embolization was made through an expert consensus conference in 5 centers.

\section{Data Analysis}

The outcomes of interest included the following: 1) angiographic and clinical characteristics, 2) rates of initial treatment failure or recurrence in neurosurgical and endovascular treatments, 3) the survival time for retreatment, 4) risk factors for treatment failure, 5) the success rate of retreatment, 6) rates of complications for the endovascular versus neurological procedures, 7) neurological outcomes, and 8) risk factors for lack of neurological improvements in gait. Angiographic data were reviewed by neuroradiologists in each center, who were blinded to the purpose of the present study, and then rereviewed by three neurosurgeons (K.T., T.E., and T.Y.). Reviewers were certified by the Neurospinal Society of Japan and had $>20$ years of experience in spinal surgery. According to the meta-analysis by Bakker et al., ${ }^{9}$ a patient with a fistula that required additional neurosurgery or endovascular treatment after the index treatment within 1 month of the initial treatment was defined as a case of initial treatment failure; a patient with recanalization of the fistula confirmed by angiography more than 1 month after the initial treatment was defined as a case of recurrence. Patients for whom more than one treatment was performed were regarded as a single case; only the index treatment was registered. Complications included CSF leaks, surgical site infection, postoperative hematoma, spinal cord infarction, vessel perforation, deep venous thrombosis, and pulmonary embolism. Complications were considered to be major if they required additional surgery or resulted in permanent neurological deficits. The neurological status was evaluated with the modified Rankin Scale (mRS) score and Aminoff-Logue (AL) gait and AL micturition grades. The AL grades for gait (range 0-5) and micturition (range 0-3) are as follows: ${ }^{4}$ gait grade $0=$ normal; $1=$ leg weakness, abnormal gait or stance, but no restriction of activity; $2=$ restricted activity but not requiring support; 3 = requiring 1 stick for walking; 4 = requiring 2 sticks, crutches, or walker; and 5 $=$ confined to wheelchair; micturition grade $0=$ normal; 1 $=$ hesitancy, urgency, or frequency; $2=$ occasional urinary incontinence or retention; and $3=$ total incontinence or retention. The neurological outcomes of patients were reviewed from medical charts or telephone interviews by the clinicians in each center, who were blinded to the purpose of the present study, between February 2019 and February 2020. 
Takai et al.

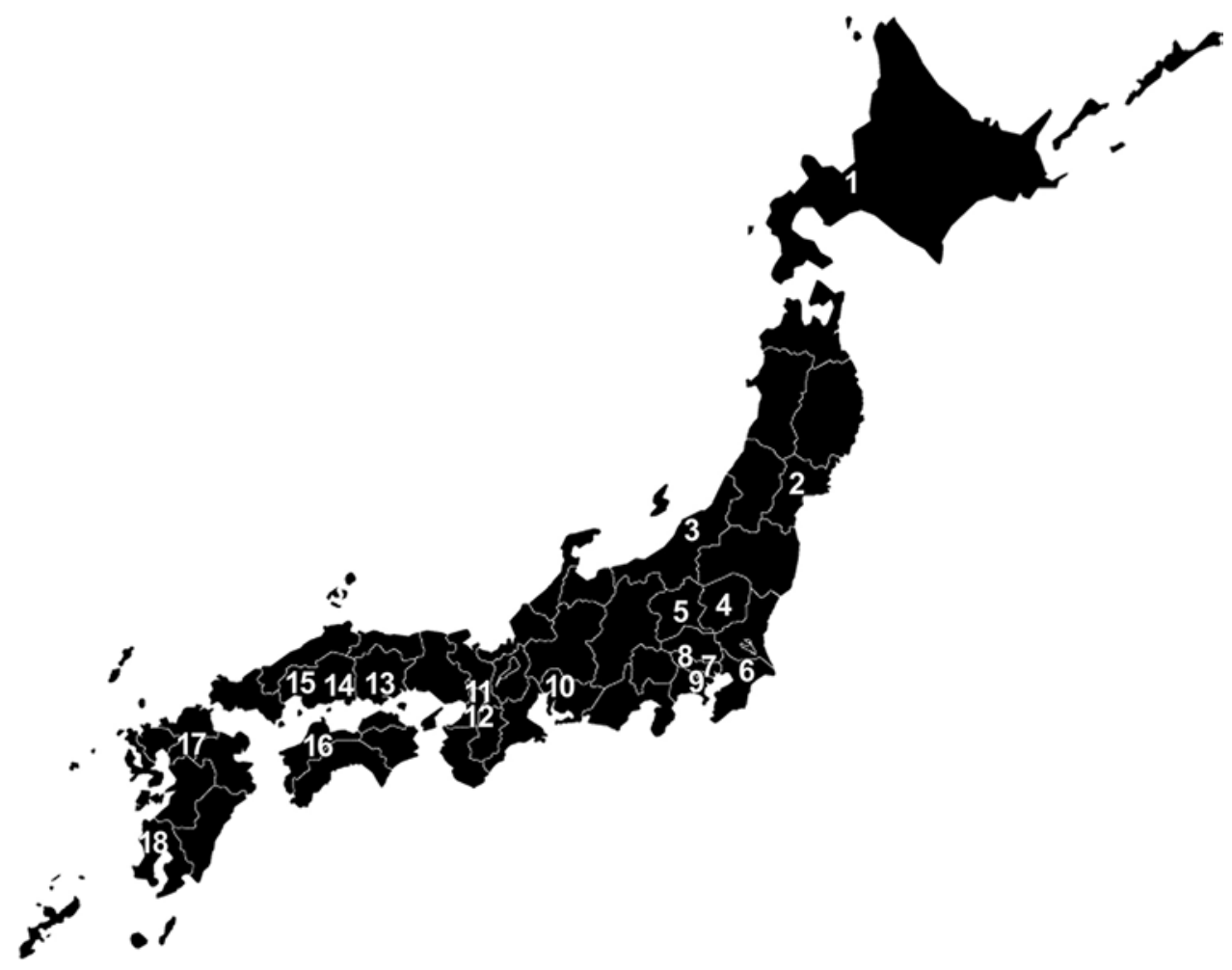

FIG. 1. Geographic distribution of 18 centers in Japan and the distribution of patients with dAVFs. 1. Department of Neurosurgery, Hokkaido University Hospital $(n=16) ; 2$. Department of Neurosurgery, Kohnan Hospital $(n=20) ; 3$. Department of Orthopedic Surgery, Niigata University Medical and Dental Hospital $(n=10) ; 4$. Department of Neurosurgery, Dokkyo Medical University Hospital $(n=11) ; 5$. Department of Neurosurgery, Gunma University Hospital $(n=13) ; 6$. Department of Orthopedic Surgery, Japanese Red Cross Narita Hospital $(n=12) ; 7$. Department of Neurosurgery, The University of Tokyo Hospital $(n=15) ; 8$. Department of Neurosurgery, Tokyo Metropolitan Neurological Hospital $(n=31) ; 9$. Department of Neurosurgery, Yokohama City University Hospital ( $n$ $=8) ; 10$. Department of Neurosurgery, Nagoya University Hospital $(n=9) ; 11$. Department of Neurosurgery, Osaka City University Hospital $(n=12) ; 12$. Department of Neurosurgery, Osaka University Hospital $(n=8) ; 13$. Department of Neurosurgery, Okayama University Hospital $(n=13) ; 14$. Department of Spinal Surgery, Brain Attack Center, Ota Memorial Hospital $(n=3) ; 15$. Department of Neurosurgery, Hiroshima University Hospital $(n=6) ; 16$. Department of Neurosurgery, Ehime University Hospital $(n=5) ; 17$. Department of Neurosurgery, Kurume University Hospital $(n=3) ; 18$. Department of Neurosurgery, Kagoshima University Hospital $(n=4)$. The map was downloaded from http://www.start-point.net for free.

\section{Statistical Analysis}

The data collected were statistically analyzed using Fisher's exact test or the chi-square test for nominal variables, the Student t-test for continuous variables, and the Mann-Whitney U-test or Wilcoxon signed-rank test for continuous variables that did not follow normal distributions or for ordinal variables. The survival time for retreatment was calculated with the Kaplan-Meier method and compared between the neurosurgical and endovascular treatment groups with a log-rank test. Risk factors for treatment failure or nonimprovement in the AL gait grade were identified via a univariate logistic regression analysis, from which variables with a $\mathrm{p}$ value $<0.1$ were selected. A multivariate logistic regression analysis was then used to identify independent risk factors for treatment failure or nonimprovement in gait after controlling simultaneously for potential confounders. The software used to conduct the analysis was SPSS Statistics version 23 (IBM Corp.). A 2-tailed $\mathrm{p}$ value $<0.05$ was considered to be significant.

\section{Results}

\section{Patients}

A total of 254 patients were registered in the present study, and 55 were subsequently excluded because of cervical lesions $(n=3)$, intradural lesions $(n=4)$, epidural lesions $(n=29)$, no angiographic data $(n=2)$, no long-term follow-up clinical data $(\mathrm{n}=10)$, and treatment before 2009 $(n=7)$. Therefore, 199 patients met the inclusion criteria.

\section{Angiographic and Clinical Characteristics}

Spinal dAVFs were frequently found in the thoracic region $(81 \%)$, fed by a single feeder $(86 \%)$, and shunted into an intradural vein via the dura mater (Table 1). The fistulous connection between feeder(s) and the intradural vein was located commonly at a single spinal level in 195 patients (98\%) and at 2 independent levels in 4 patients (2\%). Spinal dAVFs commonly occurred in elderly males (mean age 66 years) and showed venous congestive myelopathy. The mean duration of symptoms before treatment was 
Takai et al.

TABLE 1. Angiographic and clinical characteristics of dAVFs

\begin{tabular}{cccc}
\hline Characteristic & Neurosurgery $(n=145)$ & Endovascular $(n=50)$ & $p$ Value \\
\hline Angiographic findings & & & \\
\hline Thoracic fistula & $115(79 \%)$ & $43(86 \%)$ & 0.21 \\
\hline No. of feeders & $1(1-1)$ & $1(1-2)$ & $<0.001^{*}$ \\
\hline No. of drainers & $1(1-1)$ & $1(1-1)$ & $>0.99$ \\
\hline Clinical findings & & & \\
\hline Age, yrs & $66 \pm 11$ & $65 \pm 10$ & 0.57 \\
\hline Male & $123(85 \%)$ & $38(76 \%)$ & 0.19 \\
\hline Symptom duration, mos & $21 \pm 40$ & $20 \pm 22$ & 0.78 \\
\hline mRS score & $3(2-4)$ & $2(2-3)$ & 0.083 \\
\hline AL gait grade & $3(2-4)$ & $3(1.3-4)$ & 0.24 \\
\hline AL micturition grade & $2(1-2)$ & $1(0-2)$ & $0.005^{*}$ \\
\hline Pain/numbness & $134(92 \%)$ & $46(92 \%)$ & $>0.99$ \\
\hline
\end{tabular}

Values are presented as number (\%) of patients, median (IQR), or mean \pm SD unless otherwise indicated.

* Statistically significant.

20 months because misdiagnoses were common $41 \%$ of patients). A high T2 signal in the spinal cord and/or flow voids around the spinal cord were observed in most patients with dAVFs (93\%). Slight differences were observed in angiographic and clinical characteristics between the two groups: patients treated with endovascular embolization were more likely to have dAVFs fed by more than one feeding artery; patients treated with neurosurgery were more likely to have worse micturition symptoms.

\section{Initial Treatment Failure and Recurrence}

Of the 199 patients with dAVFs, 4 with multiple AVFs were excluded from the analysis of initial treatment failure or recurrence because they all required multiple independent treatments for multiple AVFs. Among 195 patients with a single AVF, 145 were treated neurosurgically and 50 endovascularly. In the neurosurgical group, initial treatment failure occurred in 1 patient $(0.69 \%)$ and no recurrence occurred. In the endovascular group, initial treatment failure occurred in 10 patients $(20 \%)$ and recurrence in $8(16 \%)$. The rate of initial treatment failure or recurrence was significantly higher in the index endovascular treatment group (neurosurgical group, 0.69\%; endovascular group, 36\%; $\mathrm{p}<0.001$ ), while operators' experience did not significantly differ among the two groups (neurosurgical group, median 19 years [range 9-37 years]; endovascular group, median 17 years [range 8-32 years]; $\mathrm{p}=0.096$ ). The rates of initial treatment failure or recurrence based on the type of decision-making employed at a given center were $1(0.81 \%)$ of 124 patients in surgery-first centers, 12 (34\%) of 35 in endovascular-first centers, and $5(28 \%)$ of 18 in consensus conference centers $(\mathrm{p}<0.001)$. A Kaplan-Meier analysis showed longer retreatment-free survival with primary neurosurgery than with endovascular treatment ( $<<0.001$; Fig. 2). The imaging modalities used to detect treatment failure differed in each center. Among the 195 patients with dAVFs, 193 (99\%) were offered postoperative MRI and clinical evaluations, while $109(56 \%)$ were offered postoperative angiography $(\mathrm{n}=$ $61)$, CT angiography $(n=19)$, or MR angiography $(n=29)$.
The percentage of angiographic follow-ups did not significantly vary between the two groups (neurosurgical group, $52 \%$; endovascular group, $66 \% ; \mathrm{p}=0.1$ ).

\section{Risk Factors for Treatment Failure}

After univariate analyses, the risk factors for initial treatment failure or recurrence were the index endovascular treatment (OR 74; $\mathrm{p}<0.001)$ and the presence of multiple feeders (OR 3.9; $\mathrm{p}=0.003)$. After the multivariate analysis, only the index endovascular treatment remained as an independent risk factor (OR 69; 95\% CI 8.7-546; $\mathrm{p}<0.001$; Table 2).

\section{Initial Treatment, Retreatment, and Complications Neurosurgery}

In 145 patients treated by neurosurgery, 109 (75\%) underwent neurosurgical interruption of the proximal part of the intradural drainer alone, while the remaining 36 patients $(25 \%)$ underwent coagulation or resection of the dura mater including the AVF as well as interruption of the intradural drainer. The mean number \pm SD of laminectomies or laminotomies performed was $1.6 \pm 0.7$. Hemilaminectomy was performed on 124 (86\%) of 145 patients. Intraoperative indocyanine green (ICG) angiography was performed on $106(73 \%)$ of 145 patients. In the neurosurgical group, 1 patient $(0.68 \%)$ required retreatment after the index surgery. In this case, accurate localization of the fistula failed in the index treatment because a single dAVF was fed by 3 feeders at different spinal levels (left T5, T6, and T7). This failure was identified on early postoperative angiography within 1 month of the index surgery, during which the patient was without neurological decline. In this case, the patient underwent the second treatment with neurosurgery, and complete obliteration of the AVF was achieved. In all 145 patients, intradural venous drainage did not recur in the followup period regardless of whether coagulation or resection of the dura mater was performed. In 145 patients treated with neurosurgery, 6 complications (4.1\%) occurred: CSF 


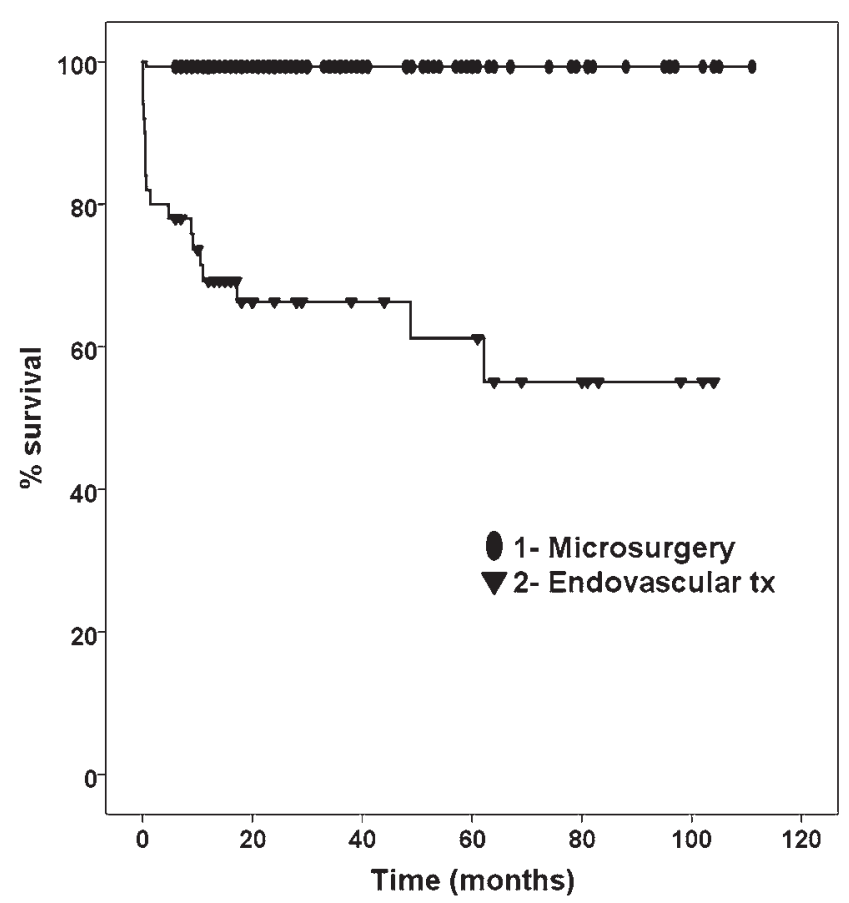

FIG. 2. Percentage survival analysis of retreatment after the index treatment by procedures. A Kaplan-Meier analysis shows longer progression-free survival with index neurosurgery than with index endovascular treatment $(p<0.001)$. $t x=$ treatment.

leak $(n=1)$, superficial wound infection $(n=1)$, postoperative worsening of paraparesis $(n=2)$, deep venous thrombosis $(\mathrm{n}=1)$, and pulmonary embolism $(\mathrm{n}=1)$. The cause of the worsening of paraparesis was considered to be venous thrombosis despite the lack of evidence on MRI. All 6 complications were attenuated by conservative management. None of these 6 patients had permanent neurological deficits associated with complications. Of the 145 patients, 143 were offered postoperative MRI in a median follow-up of 24 months (range 6-111 months). A spinal cord high T2 signal and/or flow voids decreased or disappeared in 141 (99\%) of the 143 patients.

\section{Endovascular Embolization}

In 50 patients treated with endovascular embolization,
47 underwent transarterial embolization with $N$-butyl-cyanoacrylate (NBCA) and 3 patients were treated with transarterial embolization with coils. None of the endovascular treatments involved Onyx embolic material because it has not been approved for patients with spinal AV shunts by the Pharmaceuticals and Medical Devices Agency in Japan. Of the 50 patients, 40 underwent embolization under local anesthesia and 10 under general anesthesia. None of the patients had medical comorbidities that were considered contraindications for general anesthesia. Of the 50 procedures, 10 resulted in initial treatment failure (failed catheter placement in 3; feeder occlusion in 7) and 40 in angiographic non-opacification of the fistula; however, late recurrence occurred in 8 of the 40 patients. Recurrence was observed between 5 and 62 months after the initial treatment. Of the 18 patients with initial treatment failure or recurrence, failure was identified without neurological decline in 13 patients; however, it was associated with neurological decline in the other 5. Of the 5 patients with neurological decline, treatment failure was identified on early postoperative angiography within 1 month of the index embolization in 3 patients, and it was identified on postoperative angiography 10 months after the index embolization in 2 patients. The concentration of NBCA was not associated with initial treatment failure or recurrence (complete obliteration group by a single procedure, a median of 20\%; treatment failure group, $25 \%$; $p=0.435$ ). Of the 18 patients with initial treatment failure or recurrence, 14 underwent additional neurosurgery, while 3 underwent additional endovascular embolization. The remaining patient did not receive any treatment because of the lack of a neurological decline. After the second treatment, complete obliteration of the dAVF was achieved in the 17 patients. In 50 patients treated endovascularly, 2 complications $(4.0 \%)$ occurred: neurological decline associated with spinal cord infarction due to deep NBCA migration into the intradural vein in 1 patient and vessel perforation in 1 patient. In the patient with spinal cord infarction, neurological decline (bilateral leg weakness) was ameliorated by rehabilitation. In the other patient with vessel perforation, neurological decline (right leg weakness) persisted, which was regarded as a major complication (2.0\%). The rates of total and major complications did not significantly differ between the two groups (neurosurgical group, total $4.1 \%$, major $0 \%$; endovascular group, total $4.0 \%$, major

TABLE 2. Risk factors associated with initial treatment failure or recurrence

\begin{tabular}{|c|c|c|c|c|}
\hline \multirow[b]{2}{*}{ Risk Factor } & \multicolumn{2}{|c|}{ Univariate } & \multicolumn{2}{|c|}{ Multivariate } \\
\hline & OR (95\% Cl) & $p$ Value & OR $(95 \% \mathrm{Cl})$ & $p$ Value \\
\hline Endovascular treatment & $74(9.5-577)$ & $<0.001^{*}$ & $69(8.7-546)$ & $<0.001^{*}$ \\
\hline Multiple feeders & $3.9(1.3-12)$ & $0.003^{*}$ & $1.4(0.41-4.8)$ & 0.597 \\
\hline Preop AL gait grade $\geq 4$ & $2.1(0.77-5.4)$ & 0.149 & & \\
\hline Complication & $3.0(0.58-16)$ & 0.188 & & \\
\hline Male & $0.71(0.22-2.3)$ & 0.576 & & \\
\hline Symptom duration & $1.0(0.97-1.02)$ & 0.627 & & \\
\hline Age & $0.99(0.95-1.04)$ & 0.656 & & \\
\hline
\end{tabular}


TABLE 3. Neurological outcomes

\begin{tabular}{lcll}
\hline \multicolumn{1}{c}{ Variable } & Preop & Postop & p Value \\
\hline mRS score & $3(2-4)$ & $2(1-3)$ & $<0.001^{*}$ \\
\hline AL gait grade & $3(2-4)$ & $2(1-3)$ & $<0.001^{*}$ \\
\hline AL micturition grade & $2(1-2)$ & $1(0-2)$ & $<0.001^{*}$ \\
\hline
\end{tabular}

Values are presented as median (IQR) unless otherwise indicated.

* Statistically significant.

$2.0 \% ; p=0.966, p=0.256$ ). Of the 50 patients with dAVFs treated by endovascular embolization, all 50 were offered postoperative MRI in the median follow-up of 29 months (range 6-123 months). The spinal cord high T2 signal and/ or flow voids decreased or disappeared in $49(98 \%)$ of 50 patients.

\section{Multiple dAVFs}

Among 199 patients, 4 had double independent dAVFs at different spinal levels, which were right T7 and left T12 (57 years, male); right T11 and right L2 (73 years, male); left T6 and right T8 (56 years, male); and left T6 and right T11 (84 years, male). The first 2 patients were diagnosed with double AVFs at the first angiogram, while the latter 2 were diagnosed after their neurological status did not improve after the initial treatment. Three patients underwent neurosurgery and the other patient was treated endovascularly as an initial treatment. In all patients, the initial treatment was successful for complete obliteration of the targeted fistula. Three patients underwent additional neurosurgery for the other dAVF, which led to complete obliteration of the fistula. The remaining patient underwent additional endovascular embolization for the other fistula; however, it resulted in failure because of feeder occlusion. This patient underwent a third treatment with neurosurgery and complete obliteration of the dAVF was achieved. Retreatments were performed between 1 and 8 months after the index treatment. No patients showed neurological decline.

\section{Neurological Outcomes}

In 199 patients, mRS score, AL gait and micturition grades, and pain/numbness significantly improved in the median follow-up of 26 months (range 6-123 months) af- ter the index treatment $(\mathrm{p}<0.001$, Table 3$)$. Improvements of 1 or more in mRS score or AL gait or micturition grade were observed in $111(56 \%), 121$ (61\%), and $79(40 \%)$ patients. After the multivariate analysis, the independent risk factors associated with nonimprovement in the AL gait grade were initial treatment failure or recurrence (OR 3.1; 95\% CI 1.1-8.6; Table 4) and the duration of symptoms (OR 1.02; 95\% CI 1.003-1.03). Treatment choice was not associated with postoperative nonimprovement in the gait status.

\section{Discussion}

Based on a multicenter cohort, we herein conducted a direct comparison of treatment success rates between primary neurosurgery and endovascular treatment for spinal dAVFs; primary neurosurgery was superior to endovascular treatment for complete obliteration of the fistula by a single procedure ( $99.3 \%$ vs $64 \%$ ). Endovascular treatment independently correlated with higher odds of initial treatment failure or recurrence (OR 69; 95\% CI 8.7-546; p < $0.001)$ than neurosurgery. To the best of our knowledge, the present study has the largest $(n=199)$ and most recent (2009-2018) cohort of spinal dAVFs in the literature. Only two case series studies that included more than 100 cases treated by the two procedures were previously reported in the literatue. ${ }^{5,6}$ These two studies included an old data set of patients with spinal dAVFs treated before 2009.

Due to the evolution of endovascular techniques over the last 2 decades, it has been a matter of debate whether the endovascular treatment of spinal dAVFs has caught up with and overtaken neurosurgery for the complete obliteration of fistulas. ${ }^{9}$ Another issue that remains controversial from the viewpoint of complications is whether endovascular treatment is less invasive than neurosurgery..$^{10}$ Three meta-analyses have been conducted to date: ${ }^{8-10}$ the permanent success rate of endovascular treatment for dAVFs has increased because of improvements in embolization materials from particles to glue ( $46 \%$ in $2004,72.2 \%$ in 2015 , and $69 \%$ in 2019); however, the literature favors neurosurgery over endovascular treatment because neurosurgery has a markedly higher success rate $(98 \%$ in $2004,96.6 \%$ in 2015 , and $97.2 \%$ in 2019). One of the three meta-analyses showed slightly higher complication rates with neurosurgery than with endovascular embolization (OR 1.78). ${ }^{10}$

TABLE 4. Risk factors associated with postoperative nonimprovement in the AL gait grade

\begin{tabular}{lccccc}
\hline \multirow{2}{*}{ Risk Factor } & \multicolumn{2}{c}{ Univariate } & & \multicolumn{2}{c}{ Multivariate } \\
\cline { 2 - 2 } & OR $(95 \% \mathrm{Cl})$ & $\mathrm{p} \mathrm{Value}$ & & $\mathrm{OR}(95 \% \mathrm{Cl})$ & $\mathrm{p}$ Value \\
\hline Initial treatment failure or recurrence & $2.8(1.03-7.5)$ & $0.045^{*}$ & & $3.1(1.1-8.6)$ & $0.028^{*}$ \\
\hline Symptom duration & $1.02(1.004-1.03)$ & $0.013^{*}$ & & $1.02(1.003-1.03)$ & $0.018^{*}$ \\
\hline Age & $1.03(0.99-1.1)$ & 0.066 & & $1.02(0.99-1.1)$ & 0.133 \\
\hline Preop AL gait grade $\geq 4$ & $0.63(0.34-1.2)$ & 0.141 & & \\
\hline Complication & $2.1(0.54-7.9)$ & 0.290 & & \\
\hline Male & $0.87(0.41-1.9)$ & 0.720 & & \\
\hline Endovascular treatment & $1.0(0.53-2.0)$ & 0.938 & & \\
\hline * Statistically significant. & & & &
\end{tabular}


The present study showed a higher success rate with primary neurosurgery (99.3\%) than previous meta-analyses, but a similar success rate to that of endovascular treatment (64\%). We determined the survival time for retreatment (Fig. 1) and also found that retreatment with neurosurgery had a complete obliteration rate of $100 \%$. Furthermore, the rates of complications did not significantly differ between the two groups. No patient in the neurosurgical group had major neurological complications, while one patient in the endovascular group had a permanent neurological deficit associated with a treatment-related complication.

The highest treatment success rate and low complication rate of neurosurgery in our series may be explained by advances in neurosurgical techniques over the past decade. In the conventional approach, total laminectomy has been performed to extend 1 level above and below the level of the spinal dAVF for surgical exposure of the intradural vein (3-level laminectomy). ${ }^{11,12}$ Due to advances in the accurate diagnosis of the spinal level of the fistula on neuroimaging, neurosurgery may be performed less invasively via a limited number of hemilaminectomies (1.6 \pm 0.7 levels) than the 3-level laminectomy of conventional neurosurgery. In 2009, ICG angiography that was developed for intraoperative assessment of the cerebral microcirculation was applied to spinal dAVFs. ${ }^{13,14}$ Intraoperative ICG angiography also may help localize the intradural draining vein in our series. The lack of patients endovascularly treated with Onyx embolic material is considered to be a limitation of the present study; however, a recent meta-analysis published in 2019 reported that Onyx was associated with significantly higher odds of initial failure/ late recurrence than NBCA (OR 3.87). Therefore, the exclusion of Onyx may have had a positive impact on the outcomes of endovascular treatment; however, the present results show the definite advantages of primary neurosurgical treatment over endovascular treatment for the initial failure rate and late recurrence.

Comparisons between the findings of meta-analyses and the present results showed that the success rate of endovascular treatment may have increased, but appeared to reach a plateau and was not as high as that of neurosurgery. Patients who require retreatment after endovascular treatment may not have needed retreatment if neurosurgery had been selected as the primary treatment. Therefore, we concluded that neurosurgery is still the gold standard primary treatment for patients with spinal dAVFs. However, multiple independent fistulas may be present in patients with dAVFs; if the imaging or clinical status does not improve after treatment in patients with dAVFs, multiple dAVFs need to be considered.

The present study does not deny the efficacy of endovascular treatment, because primary endovascular treatment was not an independent risk factor for postoperative nonimprovement in gait status. However, some endovascularly treated patients with recurrence showed neurological decline. We recommend a careful evaluation of the potential for success prior to endovascular embolization. The present results further emphasize the importance of an early diagnosis of treatment failure and a definite treatment before neurological decline for patients for whom endovascular embolization is selected.

\section{Study Strengths and Limitations}

The major limitation of the present study is that this was a retrospective analysis of spinal dAVFs between neurosurgery and endovascular treatment. Among the 18 centers, there may have been a selection bias in deciding the initial treatment and variations in the imaging modalities used to detect treatment failure. A prospective randomized study is needed to establish guidelines for the treatment of dAVFs. The present study included the largest and latest data set of patients with dAVFs; therefore, we consider the results obtained to be advantageous for spinal surgeons, vascular neurosurgeons, neuroendovascular interventionalists, and neuroradiologists who treat spinal dAVFs in clinical practice.

\section{Conclusions}

Neurosurgery is still the gold standard for patients with spinal dAVFs because neurosurgery via limited laminectomy was found to be superior to endovascular embolization as a primary treatment for complete obliteration of the fistula.

\section{Acknowledgments}

This study was financially supported by the Tokyo Metropolitan Government (grant number R010603007).

\section{References}

1. Takai K. Spinal arteriovenous shunts: angioarchitecture and historical changes in classification. Neurol Med Chir (Tokyo). 2017;57(7):356-365.

2. Endo T, Endo H, Sato K, et al. Surgical and endovascular treatment for spinal arteriovenous malformations. Neurol Med Chir (Tokyo). 2016;56(8):457-464.

3. Takai K, Komori T, Taniguchi M. Microvascular anatomy of spinal dural arteriovenous fistulas: arteriovenous connections and their relationships with the dura mater. J Neurosurg Spine. 2015;23(4):526-533.

4. Aminoff MJ, Logue V. The prognosis of patients with spinal vascular malformations. Brain. 1974;97(1):211-218.

5. Chibbaro S, Gory B, Marsella M, et al. Surgical management of spinal dural arteriovenous fistulas. J Clin Neurosci. 2015; 22(1):180-183.

6. Hessler C, Regelsberger J, Grzyska U, et al. Therapeutic clues in spinal dural arteriovenous fistulas-a 30 year experience of 156 cases. Cent Eur Neurosurg. 2010;71(1):8-12.

7. Lad SP, Santarelli JG, Patil CG, et al. National trends in spinal arteriovenous malformations. Neurosurg Focus. 2009; 26(1):1-5.

8. Steinmetz MP, Chow MM, Krishnaney AA, et al. Outcome after the treatment of spinal dural arteriovenous fistulae: a contemporary single-institution series and meta-analysis. Neurosurgery. 2004;55(1):77-88.

9. Bakker NA, Uyttenboogaart M, Luijckx GJJ, et al. Recurrence rates after surgical or endovascular treatment of spinal dural arteriovenous fistulas: a meta-analysis. Neurosurgery. 2015;77(1):137-144.

10. Goyal A, Cesare J, Lu VM, et al. Outcomes following surgical versus endovascular treatment of spinal dural arteriovenous fistula: a systematic review and meta-analysis. J Neurol Neurosurg Psychiatry. 2019;90(10):1139-1146.

11. Afshar JK, Doppman JL, Oldfield EH. Surgical interruption of intradural draining vein as curative treatment of spinal dural arteriovenous fistulas. J Neurosurg. 1995;82(2):196200 . 
12. Oldfield EH, Di Chiro G, Quindlen EA, et al. Successful treatment of a group of spinal cord arteriovenous malformations by interruption of dural fistula. J Neurosurg. 1983;59(6): 1019-1030.

13. Colby GP, Coon AL, Sciubba DM, et al. Intraoperative indocyanine green angiography for obliteration of a spinal dural arteriovenous fistula. J Neurosurg Spine. 2009;11(6):705-709.

14. Takai K, Kurita H, Hara T, et al. Influence of indocyanine green angiography on microsurgical treatment of spinal perimedullary arteriovenous fistulas. Neurosurg Focus. 2016; 40(3):E10.

\section{Disclosures}

The authors report no conflict of interest concerning the materials or methods used in this study or the findings specified in this paper.

\section{Author Contributions}

Conception and design: Takai. Acquisition of data: all authors. Analysis and interpretation of data: Takai, Endo, Yasuhara, Seki, K Watanabe, Y Tanaka, Kurokawa, Kanaya, Honda, Itabashi, Ishikawa, Murata, T Tanaka, Nishimura, Eguchi, Takami, Y
Watanabe, Nishida, Hiramatsu, Ohtonari, Yamaguchi, Mitsuhara, Matsui, Uchikado, Hattori, Yamahata. Drafting the article:

Takai. Critically revising the article: Endo, Yasuhara, Kurokawa.

Reviewed submitted version of manuscript: all authors. Approved the final version of the manuscript on behalf of all authors: Takai. Statistical analysis: Takai. Administrative/technical/material support: Taniguchi. Study supervision: Takai.

\section{Supplemental Information}

\section{Previous Presentations}

Portions of this work were presented in abstract form at the 34th Annual Meeting of the Neurospinal Society of Japan, Sapporo, Hokkaido, Japan, June 20, 2019.

\section{Correspondence}

Keisuke Takai: Tokyo Metropolitan Neurological Hospital, Tokyo, Japan.takai-nsu@umin.ac.jp. 